\title{
Defining and detecting emergence in complex networks
}

\author{
Fabio Boschetti ${ }^{1}$, Mikhail Prokopenko ${ }^{2}$, Ian Macreadie ${ }^{3}$, and Anne-Marie Grisogono ${ }^{4}$ \\ ${ }^{1}$ CSIRO Marine Research, Floreat, WA, Australia \\ ${ }^{2}$ CSIRO Information and Communication Technologies Centre, North Ryde, NSW, Australia \\ ${ }^{3}$ CSIRO Health Sciences and Nutrition, Parkville, Victoria, Australia \\ fabio.boschetti,mikhail.prokopenko,ian.macreadie@csiro.au \\ ${ }^{4}$ Defence Science and Technology Organisation, Edinburgh, SA, Australia \\ Anne-Marie.Grisogono@dsto.defence.gov.au
}

\begin{abstract}
Emergence is seen as the most significant feature discriminating "complex" from "non complex" systems. Nevertheless, no standard definition of emergence is currently available in the literature. This lack of a shared view affects the development of tools to detect and model emergence for both scientific and engineering applications. Here we review some definitions of emergence with the aim to describe how they can be implemented in algorithms to detect and model emergence in sensor and communication networks.
\end{abstract}

\section{Introduction}

Complex systems are often defined in the literature as ensembles of a large number of interacting simple entities, which display emergent properties. Such emergent properties are what make such systems "complex", as well as interesting to study. However, definitions of emergence available in the literature differ considerably in both their philosophical approach and formal rigour. This lack of shared understanding makes communication between scientists and practitioners in Complex System Science difficult, and, worse still, represents an obvious challenge in the development of analytical tools for the formal and experimental study of emergent properties.

There are many reasons why the study of emergence is important. The most obvious is that emergent behaviour seems to be ubiquitous in Nature. And emergence does not seem to arise solely from natural systems: Chaitin [2] shows us how emergent properties can be found also in something as simple and "human-made" as number theory. Another reason why attention is focused on the study of emergence is that standard analytical tools used in physics do not seem to be able to describe the generation of "novelty" [9]. The physics community has long aimed at the discovery of fundamental physical equations able to summarize the inner working of all aspects of Nature, which has been popularized as the quest for a "theory of everything". This led some authors to claim that the understanding of emergence is a crucial missing component in our understanding of the world [14].

Confident that the study of emergence is a worthwhile endeavour, in this review, we cover some definitions of emergence available in the literature. Our aim is not philosophical, but practical. We do not attempt to suggest a "best" definition, rather we favour definitions leading to the development of numerical/analytical tools enabling formal study and experimentation on emergent properties. Our aim is ultimately towards the many engineering applications which nowadays depend on development of intelligent self-organizing networks in 
order to tackle problems of increasing complexity and size. In these systems, the desired responses emerge by self-organization: patterns at the global level emerge solely from interactions among the lower-level components acting on rules which are executed using only local information, without reference to the global pattern. In the rest of this paper we ask the following questions:

1. what view of emergence captures the process that we expect will happen in large ensembles of lower-level components like the ones we described above?

2. how would such a view of emergence lead us in the development of useful algorithms/tools to detect and/or model the emergent processes?

\section{Some common definitions of Emergence}

Probably the most common definition of emergence is "a property is emergent if it cannot be explained from the properties and interactions of the lower level entities". Kubik [11], Shalizi [25] and Crutchfield [3] criticise this definition, mostly on the basis that such definition simply implies that we are currently unable to explain its relation to lower level entities. One day, with better scientific knowledge, we may be able to do so. Consequently such a statement is based on a temporary state of (lack of) knowledge of the observer rather than on an intrinsic property of a system (see the discussion of causation and control in Pattee, [17]).

A slightly more refined version of the above definition reads "a property is emergent if it is not displayed by the lower level entities" Kubic [11] (see also Rosen [23]: "One way to define emergence is to call a behavior of a system emergent when it can no longer be described by the model that described the system until then"). A potential problem with this definition is that basically everything can be seen as emergent according to it. For example, all macroscopic matter is made of atoms, and no individual atoms display the features of the macroscopic material [1]. Arguably, this definition has more appeal than the previous one since it offers itself to numerical analysis. A considerable body of work explores this view of emergence, in particular in the Cellular Automata (CA) literature. For example, the way intrinsic computation may emerge spontaneously and come to dominate the dynamics of physical systems when those systems are in the vicinity of a second-order phase transition has been studied by Langton [13], Mitchell et al. [16] and Wolfram [29], among others. More recently, Wiedermann and van Leeuwen [28] have explored the emergent computation capabilities of large number of interacting agents. They formally prove that their emergent computational power goes beyond traditional systems and results in super Turing computational capabilities.

More formally, Shalizi [25] proposes that "a feature is emergent if it can provide better predictability on the system behaviour, compared to the lower level entities". Importantly, Shalizi [25] and Crutchfield [3] give a formal, information-theoretic definition of the above concept as well as numerical tools to implement it. Interestingly, while the idea of predictability naturally involves an agent (observer), the "measure" of emergence they provide is observer independent, and thus an intrinsic property of a system.

We would like to point out, however, that even if such a measure is observer-independent, the observation structure/topology (e.g., the CA itself, a topology of a network, etc.) is provided a priori, not necessarily by the "observer". It can be argued that resultant patterns emerging as a result of observer-independent processes are patterns only with respect to a certain observation structure/topology, imposed on or selected in the environment. An interesting aspect, however, is that the choice of such a structure/topology is not entirely decoupled from 
the environment. In fact, this choice often depends on the emergent phenomena. So the process can be best characterised in terms of tangled hierarchies exhibiting Strange Loops: "an interaction between levels in which the top level reaches back down towards the bottom level and influences it, while at the same time being itself determined by the bottom level" [8].

This view involves the concept of "downward causation" [6,1,7]. Roughly, "a feature is emergent if it has some sort of causal power on lower level entities". While common views of emergence assume that lower level entities must have an "upward" causation on the emergent features, this approach requires a 2-way causal relation. As an example, we can imagine individuals organising into a community. Their actions affect how the community develops (upward causality) and the development of the community itself affects the behaviour and interaction of the individuals (downward causality). A critique of the concept of downward causation can be found in [17]. This definition has an obvious appeal since it clearly goes beyond the reductionist approach to the analysis of complex system. An application example of this approach is presented by Prokopenko and Wang [20], who demonstrated that self-inspection and self-repair of a sensor and communication network can be described in self-referential terms.

It is our opinion that the last three definitions are particularly suited to study multi-agent engineering systems:

1. Defining emergence as "a property which is not displayed by the lower level entities" provides us with a set of information-theoretic tools for "quick and dirty" detection of emergence.

2. The Computational Mechanics view of emergence as increased predictability on system behavior allows us to focus on a subset of features which carry maximum information about the system. Model reduction and predictability are the sought outcomes.

3. Finally, the concept of "downward causation" seems to aim at a deeper understanding of the system behaviour and its multiscale/multilevel functioning.

In the remainder of the paper we discuss potential tools implementing these ideas in multiagent systems studies.

\section{3 "Tools" to detect emergence}

Among the tools useful in an initial, exploratory investigation of the dynamics, or in the subsequent visual representation and description of the dynamics itself, we can point out the dimensionality-reduction tools such as Self-Organising Maps (SOM), Local Linear Embedding (LLE and its variants), Isomap, etc. The purpose of these tools is to identify low(er) dimensional manifolds in higher dimensional data sets. They are used in visualisation, data compression but also data mining with, potentially, useful application in the study of emergence. Under the assumption that the coordination between lower entities should constrain their behaviour, we may expect emergent features to occupy a lower dimensional state space compared to the uncoordinated dynamics.

Thus, one obvious approach to detect emergence is based on the idea that the complex behaviour of interacting components results in some form of coordination: a persistent multiagent relationship distinct from both chaotic and completely ordered dynamics. In other words, a departure from randomness, and correlations between components, may be seen as a very first step in the detection of emergent properties. It is thus natural to look at various informationtheoretic measures as a basis for the approach. 


\subsection{Emergent patterns in multi-agent systems: macro-level}

Langton has identified in his seminal work [13] an increase in the mutual information as an indication of a phase transition from "order" to "chaos" in CA. In particular, trajectories of entropy $H(A)$ and mutual information $I(A ; B)$ between a cell and itself at the next time-step were obtained while varying the parameter $\lambda-$ the ratio of cells with a given property. It was observed that the average mutual information has a distinct peak at the transition point. Similarly, a rule-space of 1-dimensional cellular automata was characterised with the Shannon entropy of rules' frequency distribution [30]. More precisely, given a rule-table (the rules that define a CA), the input-entropy at time step $t$ is defined as

$$
S^{t}=-\sum_{i=1}^{m} \frac{Q_{i}^{t}}{n} \log \frac{Q_{i}^{t}}{n},
$$

where $m$ is the number of rules, $n$ is the number of cells (system size), and $Q_{i}^{t}$ is the number of times the rule $i$ was used at time $t$ across the CA. Wuensche [30] has convincingly demonstrated that only complex dynamics exhibits high variance of input-entropy, leading to automatic classification of the rule-space. Importantly, the peak of input-entropy variance points to a phase transition as well, indicating the edge of chaos (complexity). An information-theoretic approach to quantifying information flows in agent-environment interactions was also constructively used in recent studies of perception-action loops and sensor evolution [10].

It is also well-known that graph connectivity can be analysed in terms of the size of the largest connected subgraph (LCS) and its standard deviation obtained across an ensemble of graphs, as suggested by the random graphs theory [5]. In particular, critical changes occur in connectivity of a directed graph as the number of edges increases: the size of the LCS rapidly increases as well and fills most of the graph, while the variance in the size of the LCS reaches a maximum at some critical point before decreasing. Thus, a graph-theoretic metric based on LCS variance may capture spatial aspects of desired emergent patterns (connectivity), while a metric based on entropy of local agent variables (rules, coordinates, velocities, etc.) may capture temporal persistence of emergent behaviour.

In the context of multi-agent networks it may be possible to unify the information-theoretic and graph-theoretic representations of the metrics. One feasible average measure of a complex network's heterogeneity is given by the entropy of a network defined through the link distribution. The latter can be defined via the simple degree distribution - the probability $P_{k}$ of having a node with $k$ links, or via the remaining degree distribution [26]. Similarly, one can capture the average uncertainty of the network as a whole, using the joint entropy based on the joint probability of connected pairs $P_{k, k^{\prime}}$. Ultimately, the amount of correlation between nodes in the graph can be calculated via the mutual information measure, the information transfer, as

$$
I\left(P ; P^{\prime}\right)=H(P)-H\left(P \mid P^{\prime}\right)=\sum_{k=1}^{m} \sum_{k^{\prime}=1}^{m} P_{k, k^{\prime}} \log \frac{P_{k, k^{\prime}}}{P_{k} P_{k^{\prime}}} .
$$

In general, the mutual information $I\left(P ; P^{\prime}\right)$ is a better, more generic measure of dependence: correlation functions, like the variance in the size of the LCS, "measure linear relations whereas mutual information measures the general dependence and is thus a less biased statistic" [26]. It could be argued that only complex dynamics exhibits high variance, and the peak of this variance would point to a phase transition in connectivity. 
The distinction between information-theoretic and graph-theoretic metrics is identified and put to use by Prokopenko et al. [22] in the context of self-organizing sensor and communication networks. These metrics contributed to a specification of various evolvable aspects: temporal persistence and spatial connectivity of emergent task-oriented network's sub-structures $[22,27]$.

Minati [15] proposes a measure of ergodicity to detect emergence. A system is commonly defined as ergodic when the average, at a single instant of time, on all microscopic behaviors present within the system, is equal to the time average on the behaviour of a single component. This property best describes systems at some form of stable equilibrium which offer themselves naturally to be studied via statistical mechanics tools. Since the property of ergodicity is completely lost during a phase transition, or via structural changes Minati proposes to detect increase of ergodicity as a signature of the evolution towards a new state which may be considered as emergent.

\section{Emergence and predictability: the Computational Mechanics view}

Within the Computational Mechanics school, Crutchfield [3] distinguishes pattern formation and intrinsic emergence. Intrinsic emergence refers to the emergent features which are important within the system because they confer additional functionality to the system itself, like supporting global coordination-computation-behaviour (e.g., the emergence of coordinated behaviour in a flock of birds, or in stock market pricing, allows efficient global information processing through local interaction, which benefits each of the individual components of the system).

The algorithmic approach behind the concept of Computational Mechanics [24] is proposed as the main tool in the study of intrinsic emergence, which aims to model the way information is processed within a system. Shalizi [25] formalises this approach by proposing the following definition: "A derived process is emergent if it has a greater predictive efficiency than the process it derives from". This definition is interesting since it defines emergence as a property of the system, not of the observer. Also, if offers some specific information-theoretic measures which can be implemented via the machinery of the Computational Mechanics approach. The approach is based on the Causal-State Splitting Reconstruction (CSSR) algorithm [24], which aims to reconstruct the dynamical states of a system and their transition probabilities. Via the use of the algorithm, not only the statistical complexity of the process can be measured, but emergent features (i.e., the ones which maximize predictability potential) can be automatically extracted from the process measurement. The ideas can be applied to both temporal and spatial patters. Of particular interest is the application of the above concept to the study of the evolutionary emergence of complexity in small population of simple agents and the analysis of the structural hierarchies which allow for self-organisation [4].

\section{A first step towards modelling Downward Causation: emergent patterns in multi-agent communication space (micro-level)}

Self-organisation may seem to contradict the second law of thermodynamics that captures the tendency of systems to disorder. The "paradox" has been explained in terms of multiple coupled levels of dynamic activity - the Kugler-Turvey model [12] — self-organisation and the loss of entropy occurs at the macro-level, while the system dynamics on the micro-level 
generates increasing disorder. One convincing example is described by Parunak and Brueckner [18] in the context of pheromone-based coordination. Their work defines a way to measure entropy at the macro level (agents' behaviours lead to orderly spatiotemporal patterns) and micro level (chaotic diffusion of pheromone molecules). In other words, the micro level serves as an entropy "sink" - it permits the overall system entropy to increase, while allowing selforganisation to emerge and manifest itself as coordinated multi-agent activity on the macro level. Another example relates a macro-level increase of coordination potential within a multiagent team, indicated by a macro-level decrease in epistemic entropy of agents' joint beliefs, with a micro-level increase in the entropy of the multi-agent communication space [19].

Similarly, it can be shown that the emergence of multi-agent networks, indicated by the minimal variance of their fragments (an approximation of the network heterogeneity), is explained by increased entropy on a micro-level. This micro-level is the communication space where the inter-agent messages are exchanged [21]. A characterisation of the micro-level (the entropy "sink") can be obtained if one estimates the "regularity" of the communication space. In summary, macro-level ("global-view") metrics may capture the quality of the emergent solutions in terms of observable coordination activities, while micro-level metrics may verify the solution in terms of the multi-agent communications.

\section{Conclusions}

We believe that certain basic emergent properties are shared by very different systems, and hence, steps forward in our understanding and modelling of emergence would have huge practical implication for disparate applications. With the view of studying large systems of self-organising agents, we have reviewed some definitions of emergence and described how they could lead to useful tools for the detection of emergence, for the identification of features which are maximally informative about the system dynamics (which could lead to model complexity reduction) and to the better understanding of multi-scale and multi-level information processing in complex systems.

\section{References}

1. Bickhard, M. H., 2000, Emergence. In P. B. Andersen, C. Emmeche, N. O. Finnemann, P. V. Christiansen (Eds.) Downward Causation. (322-348). Aarhus, Denmark: University of Aarhus Press.

2. Chaitin G., Meta Math!, e-book, 2004. (To be published Sept. 2005 by Pantheon Books, NY.) http://www.cs.auckland.ac.nz/CDMTCS/chaitin/omega.html

3. Crutchfield J., 1994, The Calculi of Emergence: Computation, Dynamics, and Induction, Physica D 75 1994: 11-54.

4. Crutchfield, J., and Gornerup, O., 2004, Objects That Make Objects: The Population Dynamics of Structural Complexity, Santa Fe Institute Working Paper 04-06-020. arxiv.org e-print adaporg/0406XXX.

5. Erdös, P. and Rényi, A., 1961, On the strength of connectedness of random graphs. Acta Mathematica Scientia, Hungary, 12, 261-267.

6. Goldstein, J., 2002, The Singular Nature of Emergent Levels: Suggestions for a Theory of Emergence, Nonlinear Dynamics, Psychology, and Life Sciences Vol. 6, No. 4.

7. Heylighen, F. 1991, Modelling Emergence, World Futures, Journal of General Evolution, special issue on creative evolution, G. Kampis (ed). 
8. Hofstadter, D. R., 1989, Godel, Escher, Bach: An Eternal Golden Braid. New York: Vintage Books.

9. Hooker, C., 2004, British Journal for the Philosophy of Science, September 2004, vol. 55, no. 3, pp. 435-479(45).

10. Klyubin, A. S., Polani, D., and Nehaniv, C. L., 2004, Tracking Information Flow through the Environment: Simple Cases of Stigmergy. In Proceedings of the 9th International Conference on the Simulation and Synthesis of Living Systems (ALIFE9), Boston, USA.

11. Kubic, A., 2003, Toward a Formalization of Emergence, Artificial Life, Vol. 9, Issue 1, 41-65.

12. Kugler, P.N., and Turvey, M.T., 1987 Information, Natural Law, and the Self-Assembly of Rhythmic Movement. Lawrence Erlbaum.

13. Langton, C., 1991, Life at the Edge of Chaos, Proceedings of the 2nd Conference on Artificial Life.

14. Laughlin R., and Pines, D., 2000, The Theory of Everything, Proceedings of the National Academy of Science, Vol. 97, Issue 1, 28-31.

15. Minati G., 2002, Emergence And Ergodicity: A Line Of Research, in Minati G., Pessa E. (eds.), Emergence in Complex Cognitive, Social and Biological Systems, Kluwer.

16. Mitchell, M., Crutchfield, J. P., and Das, R., 1998, Evolving cellular automata to perform computations. In T. Back, D. Fogel, and Z. Michalewicz (eds.), Hand-book of Evolutionary Computation, Oxford: Oxford University Press.

17. Pattee, H.H., 1997, Causation, Control, and the Evolution of Complexity. In P.B. Anderson, P.V Christiansen, C. Emmeche, and N.O. Finnemann (eds.) Downward Causation, in press.

18. Parunak, H.V.D., and Brueckner, S., 2001, Entropy and self-organization in multi-agent systems. In Proceedings of the 5th International Conference on Autonomous agents, Montreal.

19. Prokopenko, M., and Wang, P., 2004, Evaluating Team Performance at the Edge of Chaos. In Polani, D., Browning, B., Bonarini, A., and Yoshida, K. (eds.), RoboCup 2003: Robot Soccer World Cup VII, LNCS 3020, 89-101, Springer.

20. Prokopenko, M., and Wang, P., 2004, On Self-referential Shape Replication in Robust Aerospace Vehicles. In Proceedings of the 9th International Conference on the Simulation and Synthesis of Living Systems (ALIFE9), Boston, USA.

21. Prokopenko, M., Wang, P., Price, D., 2005, Complexity Metrics for Self-monitoring Impact Sensing Networks. Submitted to 2005 NASA/DoD Conference on Evolvable Hardware.

22. Prokopenko, M., Wang, P., Price, D. C., Valencia, P., Foreman, M., Farmer, A. J., 2005, Selforganizing Hierarchies in Sensor and Communication Networks. Accepted by Artificial Life Journal, Special Issue on Dynamic Hierarchies, to appear.

23. Rosen, R., 1985, Anticipatory Systems. Pergamon Press.

24. Shalizi, C., Shalizi, K., Crutchfield, J., 2003, An Algorithm for Pattern Discovery in Time Series, Journal of Machine Learning Research.

25. Shalizi, C., 2001, Causal Architecture, Complexity and Self-Organization in Time Series and Cellular Automata, PhD Thesis, (http://www.cscs.umich.edu/ crshalizi/thesis/).

26. Solé, R.V., and Valverde, S., 2004, Information theory of complex networks: On evolution and architectural constraints. In E. Ben-Naim, H. Frauenfelder, and Z. Toroczkai (Eds.), Complex Networks, Lecture Notes in Physics, Vol. 650, 189-210. Berlin, Germany: Springer-Verlag.

27. Wang, P., and Prokopenko, M., 2004, Evolvable recovery membranes in self-monitoring aerospace vehicles. In Proceedings of the Eighth International Conference on Simulation of Adaptive Behaviour, 509-518, Los Angeles.

28. Wiedermann, J., and van Leeuwen, J., 2003, The Emergent Computational Potential of Evolving Artificial Living Systems, AI Communications 15, 205-215.

29. Wolfram, S., 1994, Cellular Automata and Complexity: Collected Papers, Reading, MA: AddisonWesley, 1994.

30. Wuensche, A., 1999, Classifying cellular automata automatically: Finding gliders, filtering, and relating space-time patterns, attractor basins, and the Z parameter. Complexity, vol. 4, 3, 47-66. 